\title{
Two versions of the Ikaros tale
}

Members of the Ikaros protein family are important transcriptional regulators: studies in Ikaros-deficient mice have indicated a role for Ikaros (encoded by IKZF1) in lymphopoiesis, and genetic analyses in humans have linked mutations at the IKZF1 locus with B cell acute lymphoblastic leukaemia (B-ALL). Now, Schjerven et al. investigate the involvement of individual zinc finger motifs of Ikaros in its transcriptional activity.

Zinc fingers are conserved DNAbinding motifs. Ikaros family proteins have two carboxy-terminal zinc fingers, which are involved in protein dimerization and multimerization, and four amino-terminal zinc fingers that bind DNA.

Schjerven et al. generated transgenic mice that lack $I k z f 1$ exon 4 , which encodes zinc finger 1 (Ikzf1 $1^{\Delta \mathrm{F} 1 / \Delta \mathrm{F} 1}$ mice), or that lack exon 6, which encodes zinc finger 4 (Ikzf1 ${ }^{\triangle \mathrm{F} 4 / \Delta \mathrm{F} 4}$ mice). Analysis of these mice revealed that several functions of Ikaros, including the initiation of $B$ cell development, require only zinc finger 2 and zinc finger 3, whereas other functions also require either zinc finger 1 or zinc finger 4 .

$I k z f 1^{\Delta \mathrm{F} 1 / \Delta \mathrm{F} 1}$ mice lacked the full-length Ikaros protein and only expressed an alternatively spliced Ikaros isoform, whereas Ikzf1 $1^{\Delta \mathrm{F} 4 / \Delta \mathrm{F} 4}$ mice expressed mutant forms of both isoforms.
Unlike $I k z f 1^{-/-}$mice (which lack both Ikaros isoforms), Ikzf1 $1^{\Delta \mathrm{F} 1 / \Delta \mathrm{F} 1}$ and $I k z f 1^{\Delta \mathrm{F} 4 / \Delta \mathrm{F} 4}$ mice showed only a small reduction in the numbers of pre-pro-B cells. However, $I k z f 1^{\Delta \mathrm{F} 1 / \Delta \mathrm{F} 1}$ mice had substantially fewer large pre-B cells that express IgM on their cell surface compared with wild-type and $I k z f 1^{\Delta \mathrm{F} 4 / \Delta \mathrm{F} 4}$ mice. Furthermore, low numbers of small pre-B cells and immature B cells were detected in the periphery of $I k z f 1^{\Delta \mathrm{F} 1 / \Delta \mathrm{F} 1}$ mice, which indicates that zinc finger 1 has a role in the progression of B cell development. By contrast, zinc finger 4 was mainly dispensable for B cell development but was required for the development of natural killer cells and plasmacytoid dendritic cells.

Unlike B cell development, T cell development was mainly dependent on zinc finger 4: $I k z f 1^{\Delta \mathrm{F} 4 / \Delta \mathrm{F} 4}$ mice had reduced numbers of $\mathrm{CD} 4^{-} \mathrm{CD} 8^{-}$ thymocytes compared with wild-type and $I k z f 1^{\Delta \mathrm{F} 1 / \Delta \mathrm{F} 1}$ mice. Moreover, fetal $B$ cells and $T$ cells were absent from Ikzf1 $1^{\triangle \mathrm{F} 4 / \Delta \mathrm{F} 4}$ mice, which indicates that the requirement for zinc finger 4 is different in fetal and adult mice. Importantly, the absence of fetal lymphoid tissue-inducer cells in I $k z f 1^{\triangle \mathrm{F} 4 / \Delta \mathrm{F} 4}$ mice led to defective development of lymph nodes and Peyer's patches.

These findings, together with chromatin immunoprecipitation (ChIP) sequencing analyses, indicate that zinc finger 1 and zinc finger 4 help Ikaros recognize different DNA sequences. Moreover, mRNA sequencing revealed that the genes that are differentially targeted by Ikaros proteins lacking one of the two zinc finger domains are surprisingly few.

Notably, some of the genes that were selectively upregulated in I $k z f 1^{\triangle \mathrm{F} 4 / \triangle \mathrm{FF} 4}$ mice have previously been implicated in tumour invasion and metastasis. Accordingly, Ikzf $1^{\mathrm{\triangle F} 4 / \Delta \mathrm{F} 4}$ but not $I k z f 1^{\Delta \mathrm{F} 1 / \Delta \mathrm{F} 1}$ mice developed aggressive thymic lymphomas, which suggests that the tumour suppressor activity of Ikaros depends on zinc finger 4. This was confirmed using a mouse model of B-ALL, in which irradiated recipients of $I k z f 1^{\Delta \mathrm{F} 4 / \Delta \mathrm{F} 4}$ bone marrow cells that were transduced with the oncogenic tyrosine kinase BCR-ABL developed more aggressive malignancies than mice that received transduced $I k z f 1^{\Delta \mathrm{F} 1 / \Delta \mathrm{F} 1}$ bone marrow cells.

On the basis of these observations and preliminary results of mRNA sequencing, the authors propose that their model will help to delineate the gene targets of Ikaros and the mechanisms by which Ikaros contributes to B-ALL progression in humans. Maria Papatriantafyllou

ORIGINAL RESEARCH PAPER Schjerven, $\mathrm{H}$. et al. Selective regulation of lymphopoiesis and leukemogenesis by individual zinc fingers of Ikaros. Nature Immunol. 14, 1073-1083 (2013) 